\title{
Dip and strike angles method for yield line analysis of reinforced concrete slabs
}

\author{
A. K. H. Kwan* \\ The University of Hong Kong
}

Yield line analysis is a useful method for design of reinforced concrete slabs, but has been limited to slabs of simple geometry, because up to now there has been no generally applicable and fully automatic computational procedure for complex-shaped slabs. Herein, a new yield line method that can be applied to any convex polygonal-shaped slab is developed. In this method, the deflections of the slab regions divided by yield lines are measured in terms of the dip and strike angles of the slab surfaces, which can define the geometry of all kinematically admissible collapse mechanisms or yield line patterns. The external work done and the internal energy dissipation at yield lines are evaluated as functions of the dip and strike angles, and the principle of virtual work is used to determine the corresponding load factor. The final solution is obtained by minimising the load factor with respect to the dip and strike angles. A computer program based on this method has been produced. Its correctness is verified by checking against results obtained by others for simple cases, and its versatility is demonstrated by applying it to complicated slabs subjected to point, line, patch and uniformly distributed loads.

\section{Notations}

$a, b, c$ coefficients in equation of deflection slab surface

$F_{i} \quad$ magnitude of point load

$g_{i} \quad$ geometric parameter being varied to minimise $\lambda$

$\left(g_{i}\right)_{\mathrm{o}} \quad$ current value of $g_{i}$

$\Delta g_{i} \quad$ small change to be applied to $g_{i}$ to evaluate variation of $\lambda$ with $g_{i}$

$\left(\Delta g_{i}\right)^{\prime} \quad$ change to be applied to $g_{i}$ to minimise $\lambda$

$H_{i} \quad$ intensity of line load

$M_{i} \quad$ plastic moment along yield line

$m \quad$ number of line supports

$N_{\mathrm{F}} \quad$ number of point load

$N_{\mathrm{H}} \quad$ number of line load

$N_{\mathrm{P}} \quad$ number of patch load

$N_{\mathrm{S}} \quad$ number of negative yield lines at supported edges

$N_{\mathrm{Y}} \quad$ number of positive yield lines within the slab

$n \quad$ number of point supports

$P_{i} \quad$ intensity of patch load

* Department of Civil Engineering, The University of Hong Kong, Pokfulam, Hong Kong.

(MCR 31227) Paper received 22 December 2003; last revised 21 May 2004; accepted 26 July 2004

$\begin{array}{ll}U & \text { intensity of uniformly distributed load } \\ W_{\text {ext }} & \text { external work done } \\ W_{\text {int }} & \text { internal energy dissipation along yield lines } \\ z & \text { vertical displacement of slab surface } \\ \theta & \text { dip angle } \\ \lambda & \text { load factor } \\ \lambda_{\mathrm{o}} & \text { current value of } \lambda \\ \lambda_{1} & \text { value of } \lambda \text { when of } g_{i}=\left(g_{i}\right)_{\mathrm{o}}-\Delta g_{i} \\ \lambda_{2} & \text { value of } \lambda \text { when of } g_{i}=\left(g_{i}\right)_{\mathrm{o}}+\Delta g_{i} \\ \phi & \text { strike angle } \\ \psi & \text { plastic rotation angle at yield line }\end{array}$

\section{Introduction}

There are several commonly adopted analytical methods for design of reinforced concrete (RC) slabs. For elastic design the finite-element method or grillage method may be used, whereas for plastic design the yield line method or strip method may be employed. ${ }^{1,2}$ Among these, as RC design is nowadays based mostly on the limit state design philosophy, the yield line and strip methods, which give ultimate loads or load factors, are generally preferred. However, these two design methods have been limited to relatively simple cases because of their reliance on assumed yield line or strip patterns, which up to now have had to be determined on a case-by-case basis by means of engineering judge- 
ment. Generalised analytical or numerical procedures applicable to RC slabs with complex geometry are still lacking, and consequently computerisation of the yield line and strip methods has proved to be a formidable task. To resolve the problem, the author is pointing out in this paper a new direction for the development of a generalised numerical procedure for the yield line method that may be applied to complex-shaped RC slabs.

The yield line theory was actually developed long before the classical limit state design philosophy appeared in the 1950s. Two alternative forms had been developed, one by Ingerslev $^{3}$ and the other by Johansen. ${ }^{4}$ Both forms lead to identical solutions, and have been demonstrated to be equivalent to each other. ${ }^{5}$ In either form, the yield line theory is based on assumed collapse mechanisms, each defined by a pattern of yield lines along which the slab has reached its plastic moment capacity. It is generally assumed that the slab is rigid-plastic in the sense that elastic deformation is relatively small and negligible, whereas plastic deformation can be very large because of nearperfect plasticity. Owing to the implicit assumption of near-perfect plasticity, the theory is applicable only to under-reinforced concrete slabs. For each assumed collapse mechanism, the corresponding ultimate load is evaluated using the principle of virtual work. By considering all kinematically admissible collapse mechanisms, the smallest ultimate load is taken as the final solution. Such a solution is theoretically an upperbound solution, but in practice many investigators have shown that the yield line theory is a conservative method for slabs with less than $1 \%$ steel reinforcement in each layer when failure is due to bending. ${ }^{6}$

Traditionally, yield line analysis starts by assuming yield line patterns that are kinematically admissible, and then proceeds by evaluating the corresponding ultimate load of each assumed yield line pattern. While ascertaining whether a yield line pattern is kinematically admissible, it should be noted that a yield line is actually an intersection line between two adjacent slab regions, each assumed to be rigid and rotating about an axis of rotation. For a yield line pattern to be kinematically admissible, it has to satisfy the following kinematic conditions.

(a) Every yield line or its extension must pass through the intersection point of the axes of rotation of the two adjacent slab regions.

(b) Every line support (supported edge) is an axis of rotation, and every point support (column) must lie on an axis of rotation.

(c) Every yield line is a straight line ending either at a nodal point where other yield lines meet or at a free edge. Yield lines are not allowed to intersect each other, but their ends may meet at common nodal points.

A yield line pattern is usually defined in terms of the positions, lengths and angles of the yield lines. However, as shown in Fig. 1, even for a simple rectangular slab there could be different yield line patterns, which bear no resemblance to each other. Each yield line pattern has to be defined by a separate set of geometric parameters (for example, in Fig. 1, pattern A may be defined by $\alpha_{1}, \alpha_{2}$ and $\alpha_{3}$, and pattern $\mathrm{B}$ by $\beta_{1}, \beta_{2}$ and $\left.\beta_{3}\right)$. Up to now, there has been no way of defining all the kinematically admissible yield line patterns by a single set of geometric parameters.

Since the 1970s many researchers have attempted to computerise the yield line method. However, although the degree of automation has increased over the years, most computational procedures developed ${ }^{7-9}$ are still based on assumed yield line patterns that are to be input by the user. One common way of inputting a yield line pattern is to enter the coordinates of the nodal points where yield lines meet or end. ${ }^{8,9}$ As the nodal points are related to each other by the kinematic conditions, they are not independent. If the coordinates were not input correctly, the kinematic conditions would not be satisfied. Some computer programs ${ }^{8}$ provide routines for checking the kinematic conditions, but most ${ }^{7,9}$ just rely on the user to ensure that the kinematic conditions are satisfied. So far, there has been no simple way of specifying the geometric parameters of a yield line pattern that would always satisfy the kinematic conditions. ${ }^{10}$ There is also no generally applicable rule for generating all the yield line patterns to be considered in the analysis.

To overcome the above problem, Munro and Da Fonseca $^{11}$ developed in 1978 a yield line method that does not require the input of assumed yield line patterns. In their method, no yield line pattern needs to be input or generated. Instead, a finite-element mesh is generated, and the linear programming technique is employed to search for the yield line pattern, which must follow the fixed and discrete element boundaries. When the finite-element mesh contains the actual yield lines of the collapse mechanism, the solution obtained from this discrete model coincides with that from the continuous model (the conventional model that permits continuous variation of the yield line pattern). The finite-element mesh need not be fine, but, if it does not contain the actual yield lines, the solution may not be reliable. Jennings et al. ${ }^{12}$ had attempted to resolve this problem by allowing the nodes of the finite-element mesh to adjust their positions through geometric optimisation so that hopefully the element boundaries would eventually contain the yield lines of the collapse mechanism. However, the geometric optimisation may run into convergence problems because of the presence of slope discontinuities in the optimisation function. Johnson ${ }^{13,14}$ adopted a similar approach, and had developed a strategy of first identifying the critical yield line pattern by means of a fine-mesh arrangement, which is not subjected to geometry optimisation, and then applying geometric optimisation to a simplified net, which is 


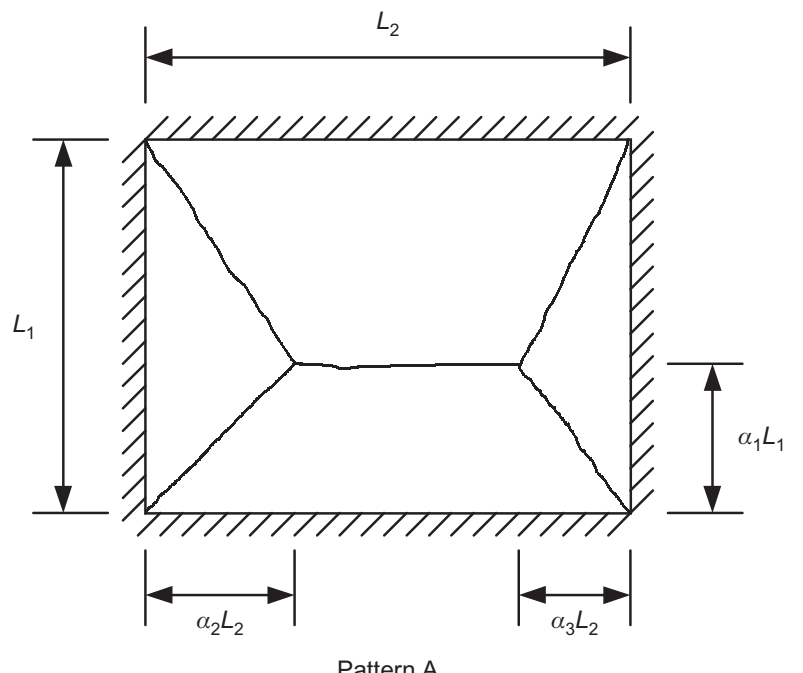

Pattern A

(a)

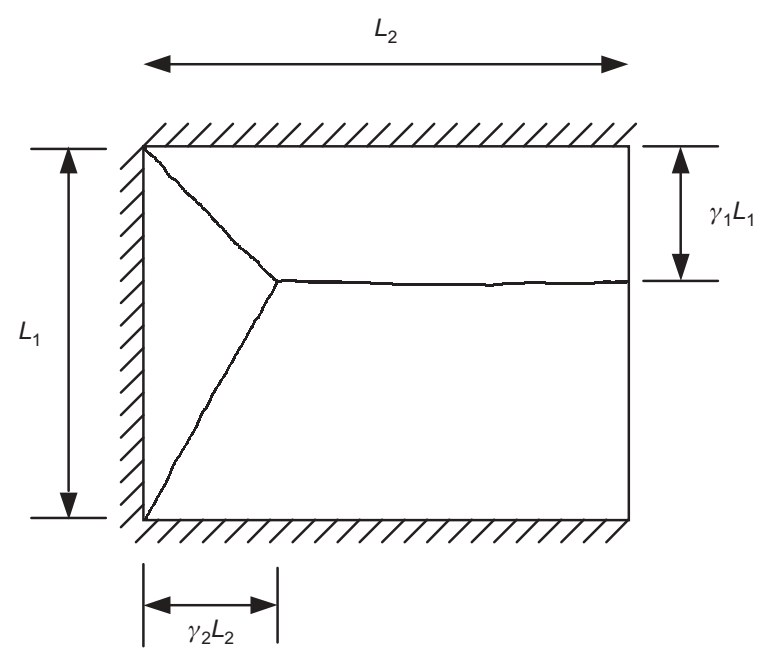

Pattern C
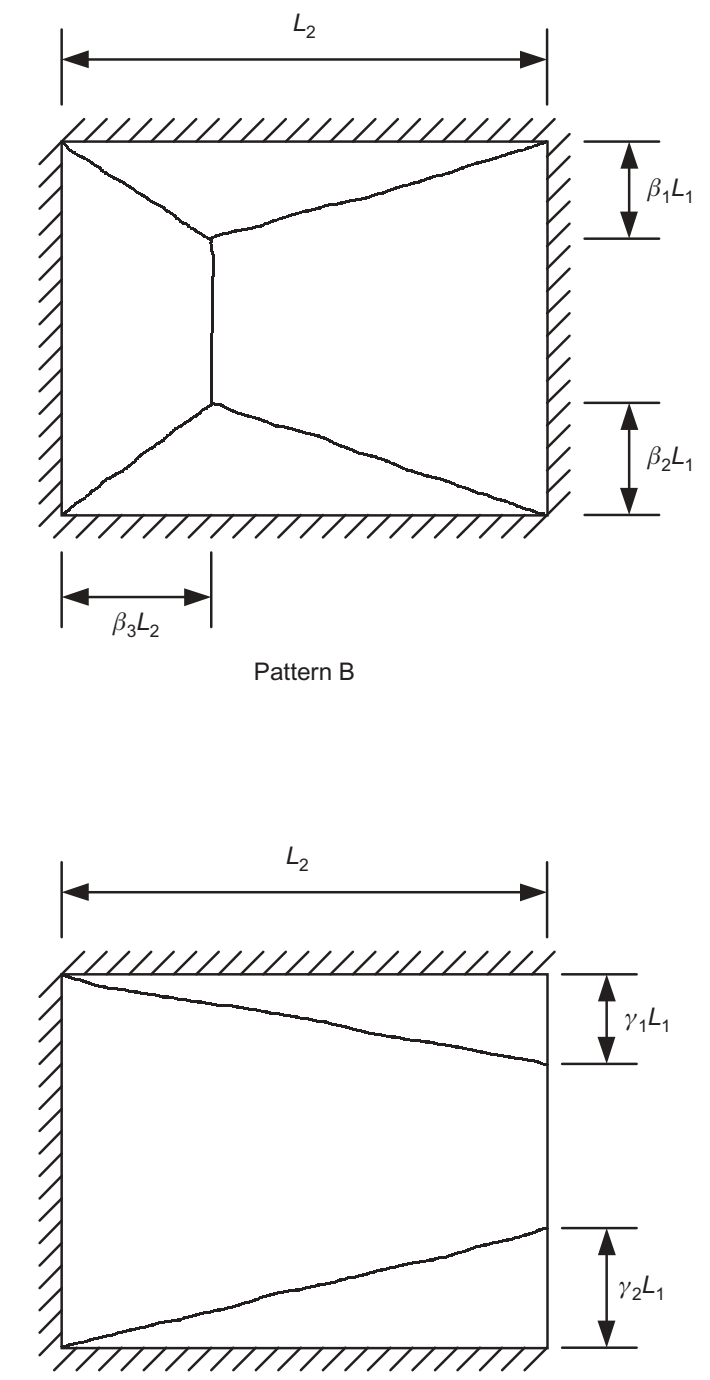

Pattern D

(b)

Fig. 1. Possible yield line patterns in a rectangular slab: (a) case 1, slab supported on all four edges; (b) case 2, slab supported on three edges only

established from the critical yield line pattern determined initially. Later, Thavalingam et al. ${ }^{15,16}$ improved Jennings et al.'s geometric optimisation technique by using the conjugate gradient method to tackle the problems due to slope discontinuities. However, some expertise is still needed by the user in knowing what topological configurations should be investigated for any particular slab.

In this paper, a new method based on the continuous model is developed. It is the author's view that the main hurdle in the computerisation of the yield line method is the generation of all kinematically admissible yield line patterns using a single set of geometric parameters. Herein, it is proposed to remove this hurdle by defining the yield line patterns in terms of the dip and strike angles of the deflected slab surfaces instead of the positions, lengths and angles of the yield lines.

\section{Dip and strike angles}

After yielding, the yield lines formed will divide the slab into separate regions bounded by the edges of the slab and the yield lines. It is postulated herein that, for a convex polygonal-shaped slab with no internal column support, each slab region is associated with either a line support (a simply supported or clamped edge) or a point support (a column at a corner between two free edges) and has an axis of rotation, as illustrated in Fig. 2. For a slab region associated with a line support, its 


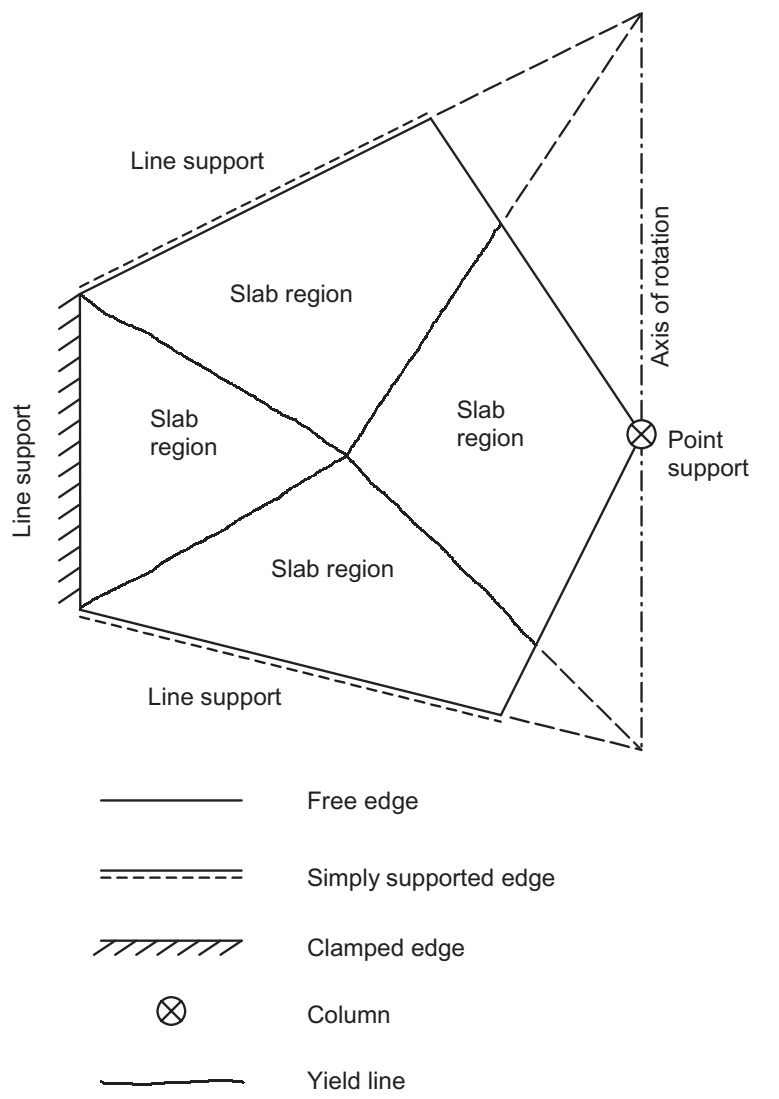

Fig. 2. Slab regions associated with line or point supports

axis of rotation is the same as the line support, whereas for a slab region associated with a point support, its axis of rotation is unknown but must pass through the point support. The axes of rotation, together with the angles of rotation of the slab regions, control the deflection mode of the slab. There is a one-to-one correspondence between the yield line pattern and the deflection mode. For any given kinematically admissible yield line pattern, the deflection mode may be determined by considering the kinematic conditions. On the other hand, for any given deflection mode, the yield line pattern may be identified from the intersection lines of the deflected slab surfaces. Hence the deflection mode of the slab may be used to define any kinematically admissible yield line pattern of the slab. It is noteworthy at this stage of theoretical development that, in 1996, Jennings ${ }^{17}$ had proved the theorem that, for a convex polygonal-shaped slab supported on all edges and with no internal column support, the yield lines will divide the slab into regions, each associated with a supported edge. Hence the above postulation may be considered an extension of Jennings' theorem.

The deflection of each slab region, which remains a plane surface because of negligible elastic deformation, may be defined in terms of its angle of rotation and the orientation of its axis of rotation. In the field of geology, an inclined stratum is mapped in terms of its dip and strike angles, ${ }^{18}$ as depicted in Fig. 3. The dip angle is the maximum slope of the stratum (same as the angle of rotation), and the strike angle is the bearing of a horizontal line on the stratum (same as the orientation of the axis of rotation). Borrowing these terminologies, the deflection of each slab region is defined in terms of its dip angle (angle of rotation) and strike angle (orientation of axis of rotation). For a slab region associated with a line support, its strike angle is predetermined because its axis of rotation must be the same as the line support, but, for a slab region associated with a point support, its strike angle is an unknown parameter to be determined.

For each line support there is a slab region associated with it, and as the strike angle is predetermined, only one unknown geometric parameter-the dip angle-is needed to define the deflection of the slab region. Likewise, for each point support, there is a slab region associated with it, but two unknown geometric parameters - the dip and strike angles - are needed to define the deflection of the slab region. If there are $m$ line supports and $n$ point supports, a total of $(m+n)$ dip angles and $n$ strike angles will be needed to define the deflection mode of the slab. As yield lines are just intersection lines between deflection slab surfaces, these geometric parameters can also uniquely define the yield line pattern. By varying the values of these geometric parameters, the whole range of yield line patterns can be generated. Moreover, the yield line patterns so generated are always kinematically admissible. In other words, this single set of geometric parameters comprising $(m+n)$ dip angles and $n$ strike angles is capable of generating all kinematically admissible yield line patterns. With these geometric parameters used to define the yield line patterns and treated as the unknowns to be solved, the main hurdle in the computerisation of the yield line method can now be removed.

\section{Generation of yield line pattern}

Yield lines are just intersection lines between deflected slab surfaces. Their positions, lengths and angles may be determined if the equations of the deflected slab surfaces are known. For a slab region associated with a line support passing through $\left(x_{1}, y_{1}\right)$ or a point support at $\left(x_{1}, y_{1}\right)$ and having dip and strike angles of $\theta$ and $\phi$ (see Fig. 3 for sign convention), the equation of its deflected surface may be derived as

$$
z=-\theta \sin \phi\left(x-x_{1}\right)+\theta \cos \phi\left(y-y_{1}\right)
$$

in which $z$ is the vertical displacement (downwards positive). Note that, as the deflected surface is a plane surface, $z$ is a linear function of $x$ and $y$. Repeating the above procedure for every slab region, the equations of all the deflected slab surfaces may be evaluated. They are generally of the form $z=a x+b y+c$.

Let a yield line be the intersection line between the 


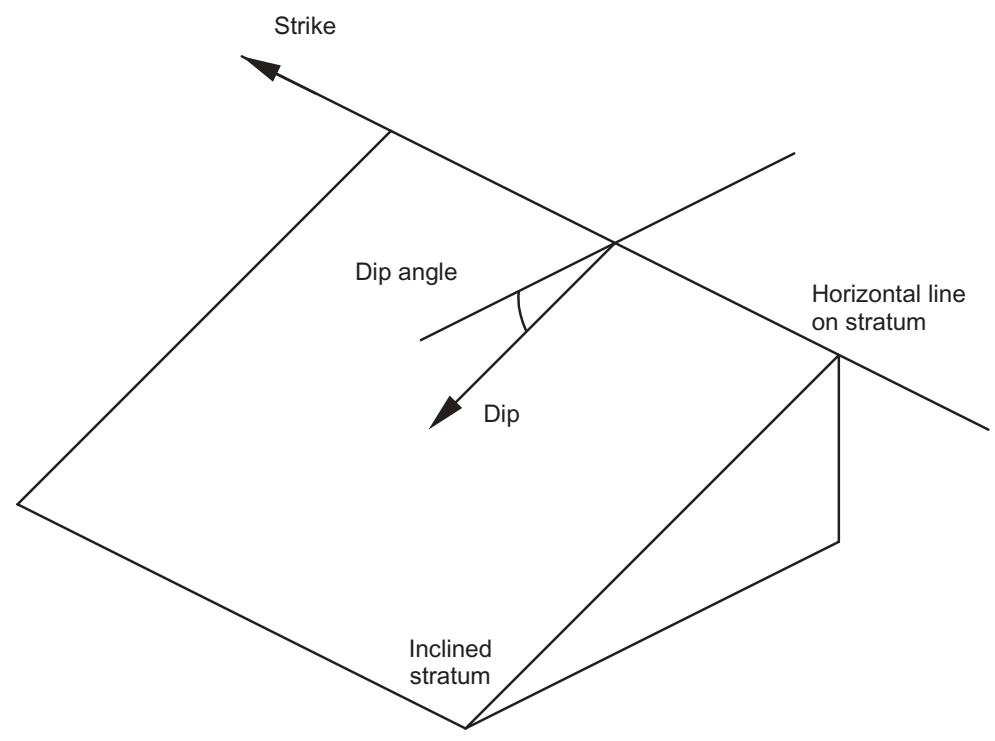

(a)
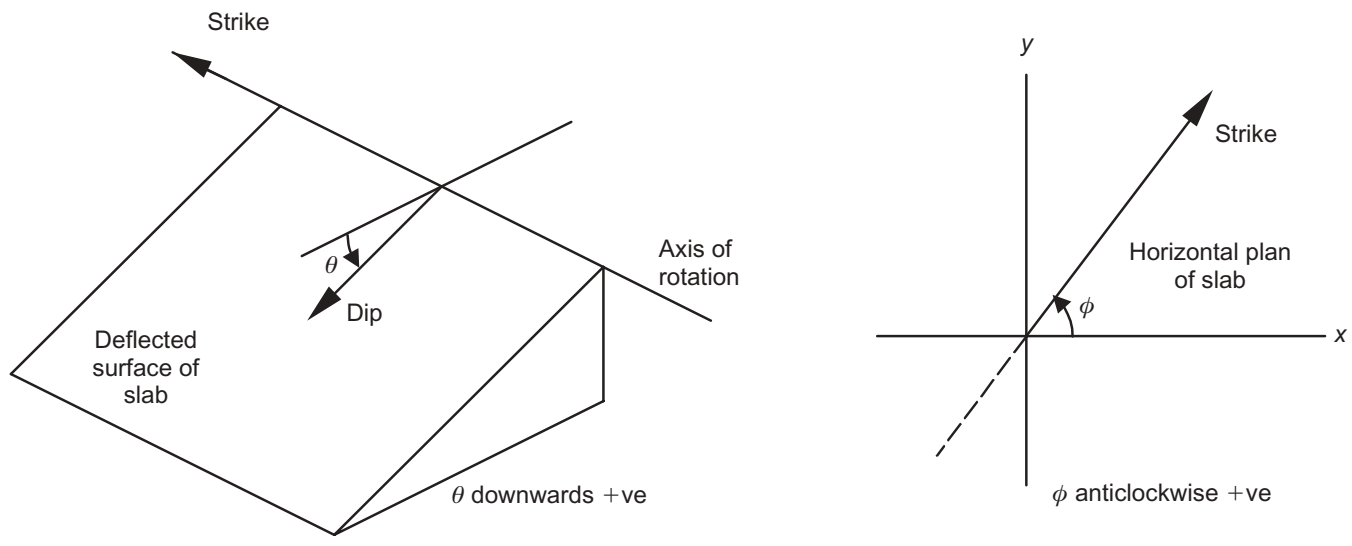

(b)

Fig. 3. Dip and strike angles: (a) mapping of an inclined stratum in geology; (b) sign convention for dip and strike angles

deflected slab surfaces of the $i$ th and $j$ th slab regions, whose equations are given respectively by

$$
\begin{aligned}
& z=a_{i} x+b_{i} y+c_{i} \\
& z=a_{j} x+b_{j} y+c_{j}
\end{aligned}
$$

Equating the vertical displacements of the two deflected slab surfaces, the equation of the intersection line may be derived as

$$
\left(a_{i}-a_{j}\right) x+\left(b_{i}-b_{j}\right) y+\left(c_{i}-c_{j}\right)=0
$$

For every pair of slab regions, one such intersection line may be obtained. However, not all intersection lines would materialise as yield lines. Moreover, intersection lines as given by the above equation are infinitely long, whereas in reality yield lines have only finite lengths. Therefore a special numerical procedure is needed to pick up the yield lines from the set of intersection lines, as explained below.

The yield line pattern is generated by considering each slab region in turn. Let the slab region being considered be the $i$ th slab region. When the deflected surface of the $i$ th slab region intersects the deflected surface of the $j$ th slab region, an intersection line as given by equation (4) is generated. At the beginning, this intersection line is infinitely long. The points where this intersection line crosses the slab boundary are then evaluated. Let the two points where the intersection line crosses the slab boundary be P and Q and their coordinates be $\left(x_{\mathrm{p}}, y_{\mathrm{p}}\right)$ and $\left(x_{\mathrm{q}}, y_{\mathrm{q}}\right)$ respectively. As only the segment of the intersection line within the slab area is relevant, the intersection line is cut to have a finite length with end points $\mathrm{P}$ and $\mathrm{Q}$. The $z$-coordinates of $\mathrm{P}$ and $\mathrm{Q}$ are then evaluated using equations (2) or (3). Denoting the $z$-coordinates of P and Q by $z_{\mathrm{p}}$ and 
$z_{\mathrm{q}}$, the coordinates of $\mathrm{P}$ and $\mathrm{Q}$ in space are obtained as $\left(x_{\mathrm{p}}, y_{\mathrm{p}}, z_{\mathrm{p}}\right)$ and $\left(x_{\mathrm{q}}, y_{\mathrm{q}}, z_{\mathrm{q}}\right)$, respectively. The line PQ cuts through the deflected surfaces of the other slab regions (slab regions other than the $i$ th and $j$ th slab regions). The parts of PQ that fall below any of the deflected surfaces of the other slab regions are then trimmed off, as they would not materialise as yield lines. The remaining part of PQ (denoted by RS, where $\mathrm{R}$ and $\mathrm{S}$ are the new end points), which stays above all of the deflected surfaces of the other slab regions, would become a yield line, whereas the end points $\mathrm{R}$ and $\mathrm{S}$ of the yield line would become nodal points of the yield line pattern.

Considering the $i$ th slab region, all the yield lines lying on its boundaries can be found by working out the intersections between its deflected surface and the deflected surfaces of all the other slab regions (slab regions other than the $i$ th slab region) and trimming off those parts of the intersection lines that would not materialise as yield lines. Repeating this procedure for each slab region in turn until all slab regions have been considered, the whole yield line pattern can be generated. The yield line pattern generated consists of the three-dimensional coordinates (i.e. $x, y$ and $z$ ) of the nodal points where yield lines meet or end and the vertical displacement everywhere in the slab (as given by the equations of the deflected slab surfaces). A flowchart of the procedure is presented in Fig. 4.

\section{Virtual work equations}

The principle of virtual work is employed to evaluate the load factor of the slab under the given loading.

For the external loads, point load, line load, patch load and uniformly distributed load are considered. Their work done, $W_{\text {ext }}$, is given by

$$
\begin{aligned}
W_{\text {ext }}= & \sum_{i=1}^{N_{\mathrm{F}}}\left(F_{i} z_{i}\right)+\sum_{i=1}^{N_{\mathrm{L}}}\left[\int H_{i} z(x, y) \mathrm{d} s\right] \\
& +\sum_{i=1}^{N_{\mathrm{P}}}\left[\int P_{i} z(x, y) \mathrm{d} A\right]+\int U z(x, y) \mathrm{d} A
\end{aligned}
$$

where $F_{i}$ is the magnitude of the point load; $H_{i}$ is the intensity per unit length of the line load; $P_{i}$ is the intensity per unit area of the patch load; $U$ is the intensity per unit area of the uniformly distributed load; $\mathrm{d} s$ is the differential length along the line load being considered; $\mathrm{d} A$ is the differential area within the patch load or uniformly distributed load being considered; and $N_{\mathrm{F}}, N_{\mathrm{H}}$ and $N_{\mathrm{P}}$ are the numbers of point load, line load and patch load, respectively. When evaluating the work done by the point load $F_{i}$, it is first necessary to determine which slab region the point load is acting on. Having determined the slab region that the point load is acting on, the vertical displacement $z_{i}$ may then be evaluated using the equation of its deflected surface.

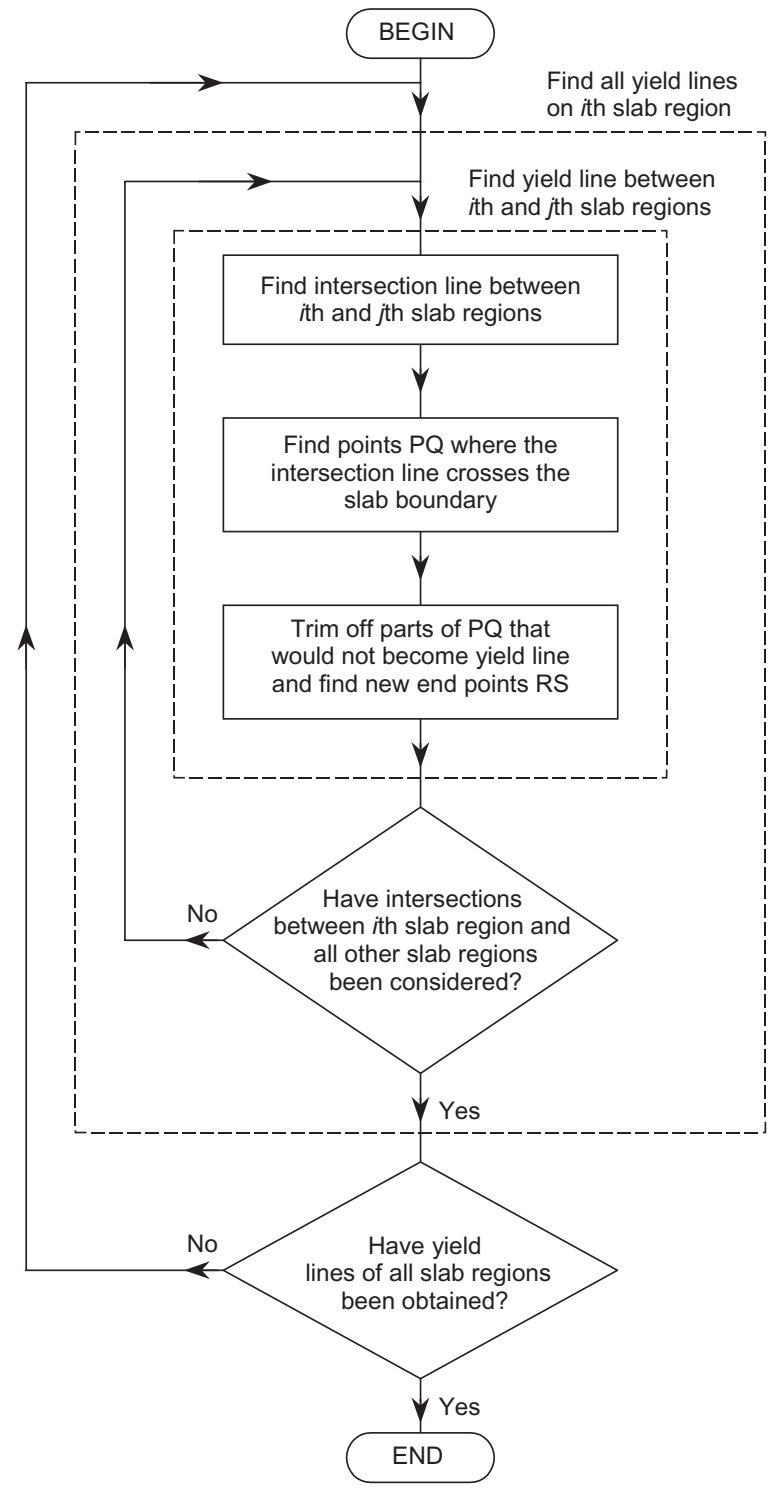

Fig. 4. Procedure for finding yield lines

Likewise, when evaluating the work done by the line load $H_{i}$, it is first necessary to determine the slab regions that the line load is acting on. The line load is then separated into several parts, each acting on one slab region. Afterwards, the work done on each slab region is obtained by integration using the equation of deflected surface of the slab region involved. The total work done by the line load is then determined by summing the work done on each slab region. Similarly, when evaluating the work done by the patch load $P_{i}$, it is first necessary to determine the slab regions that the patch load is acting on. The patch load is then separated into several parts, each acting on one slab region. After obtaining the work done on each slab region by integration, the total work done by the patch load is then determined by summing the work done on each slab region.

On the other hand, the internal energy dissipation along the yield lines, $W_{\text {int }}$, is given by

Magazine of Concrete Research, 2004, 56, No. 8 


$$
W_{\text {int }}=\sum_{i=1}^{N_{\mathrm{S}}}\left(\int M_{i} \psi_{i} \mathrm{~d} l\right)+\sum_{i=1}^{N_{\mathrm{Y}}}\left(\int M_{i} \psi_{i} \mathrm{~d} l\right)
$$

in which the first term on the right-hand side is the energy dissipation along negative yield lines at supported edges, and the second term is the energy dissipation along positive yield lines within the slab; $M_{i}$ is the plastic moment per unit length along the yield line; $\psi_{i}$ is the plastic rotation angle at the yield line; $\mathrm{d} l$ is the differential length along the yield line being considered; $N_{\mathrm{S}}$ is the number of negative yield lines at supported edges; and $N_{Y}$ is the number of positive yield lines within the slab. For a negative yield line at a supported edge, the plastic rotation angle $\psi$ is the same as the dip angle. For a positive yield line with end points $\mathrm{R}$ and $\mathrm{S}$ formed between the $i$ th and $j$ th slab regions, the plastic rotation angle $\psi$ can be shown to be given by

$$
\psi=\frac{\left(a_{i}-a_{j}\right)\left(y_{\mathrm{r}}-y_{\mathrm{s}}\right)-\left(b_{i}-b_{j}\right)\left(x_{\mathrm{r}}-x_{\mathrm{s}}\right)}{\sqrt{\left(x_{\mathrm{r}}-x_{\mathrm{s}}\right)^{2}+\left(y_{\mathrm{r}}-y_{\mathrm{s}}\right)^{2}}}
$$

in which $x_{\mathrm{r}}$ and $y_{\mathrm{r}}$ are the coordinates of $\mathrm{R}$, and $x_{\mathrm{s}}$ and $y_{\mathrm{s}}$ are the coordinates of $\mathrm{S}$.

Having obtained the external work done, and the internal energy dissipation along yield lines, the load factor $\lambda$ may be evaluated as

$$
\lambda=\frac{W_{\text {int }}}{W_{\text {ext }}}
$$

As both $W_{\text {ext }}$ and $W_{\text {int }}$ are dependent on the dip and strike angles of the slab regions, $\lambda$ is a function of the dip and strike angles.

\section{Determination of minimum load factor}

The minimum load factor is obtained by minimising $\lambda$ with respect to the dip and strike angles. Because of slope discontinuities, the minimisation procedure cannot be carried out using any numerical method that relies on the differential coefficients of $\lambda$ with respect to the variables. To overcome this difficulty, a successive parabolic minimisation technique, as explained below, is employed.

The $\lambda$-function is minimised with respect to each geometric parameter - that is, each dip or strike angle -in turn. If there are $m$ line supports and $n$ point supports, there will be a total of $(m+2 n)$ geometric parameters, namely $(m+n)$ dip angles and $n$ strike angles, that are to be varied to yield the minimum value of $\lambda$. These geometric parameters are first assigned initial values. For each dip angle, an initial value of 1.0 is assigned, and for each strike angle, an initial value equal to the mean of the bearing angles (measured anticlockwise from the $x$-axis) of the two adjacent free edges is assigned. After assigning initial values, the geometric parameters are varied in turn to minimise $\lambda$.
Let the geometric parameter being considered be denoted by $g_{i}$, and the current values of $g_{i}$ and $\lambda$ be denoted by $\left(g_{i}\right)_{0}$ and $\lambda_{0}$, respectively. The effect of a small variation of $g_{i}$ on the value of $\lambda$ is then analysed by applying small changes of $\pm \Delta g_{i}$ to $g_{i}$ and evaluating the corresponding values of $\lambda$ after the value of $g_{i}$ is changed. Let the value of $\lambda$ when $g_{i}=\left(g_{i}\right)_{\mathrm{o}}-\Delta g_{i}$ be denoted by $\lambda_{1}$, and the value of $\lambda$ when $g_{i}=\left(g_{i}\right)_{\mathrm{o}}+$ $\Delta g_{i}$ be denoted by $\lambda_{2}$. A parabolic curve is then fitted to approximate the variation of $\lambda$ with $g_{i}$, as shown in Fig. 5. From the parabolic curve, the change $\left(\Delta g_{i}\right)^{\prime}$ to be applied to $g_{i}$ that will yield a minimum value of $\lambda$ may be derived as

$$
\left(\Delta g_{i}\right)^{\prime}=\frac{1}{2}\left(\frac{\lambda_{1}-\lambda_{2}}{\lambda_{1}-2 \lambda_{0}+\lambda_{2}}\right)\left(\Delta g_{i}\right)
$$

This equation for $\left(\Delta g_{i}\right)^{\prime}$ may be applied even when there is slope discontinuity. When the magnitude of $\left(\Delta g_{i}\right)^{\prime}$ is greater than $5 \Delta g_{i}$ its magnitude is reduced to $5 \Delta g_{i}$, in order to avoid numerical instability due to excessive extrapolation. On the other hand, when the magnitude of $\left(\Delta g_{i}\right)^{\prime}$ is less than $0 \cdot 5 \Delta g_{i}, \Delta g_{i}$ is halved and the above procedure is repeated to have a new value of $\left(\Delta g_{i}\right)^{\prime}$ evaluated. After settling with the value of $\left(\Delta g_{i}\right)^{\prime}$, the change $\left(\Delta g_{i}\right)^{\prime}$ is applied to $g_{i}$ and the updated value of $\lambda$ is evaluated. If the updated value of $\lambda$ is not smaller than $\lambda_{0}, \Delta g_{i}$ is further halved and the procedure is repeated until the new value of $\lambda$ is smaller than $\lambda_{\mathrm{o}}$.

Having minimised the $\lambda$-function with respect to $g_{i}$, as outlined above, the $\lambda$-function is then minimised with respect to the other geometric parameters in turn until it has been minimised with respect to all the geometric parameters. This completes one cycle of minimisation. The minimisation cycle is repeated until the change in $\lambda$ after one minimisation cycle is less than $0 \cdot 1 \%$ or any acceptable error in load factor. $\mathrm{Nu}$ merical trials indicated that this minimisation procedure is stable and convergent. Although in some cases up to 50 minimisation cycles may be required, depending on the complexity of the slab and how close the initial values of the geometric parameters are to the correct values, the actual computer time is generally less than

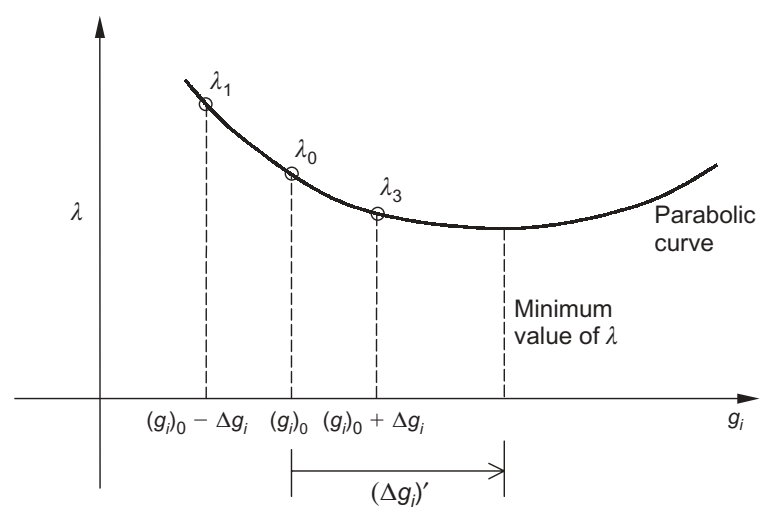

Fig. 5. Parabolic approximation of variation of $\lambda$ with $g_{i}$ 


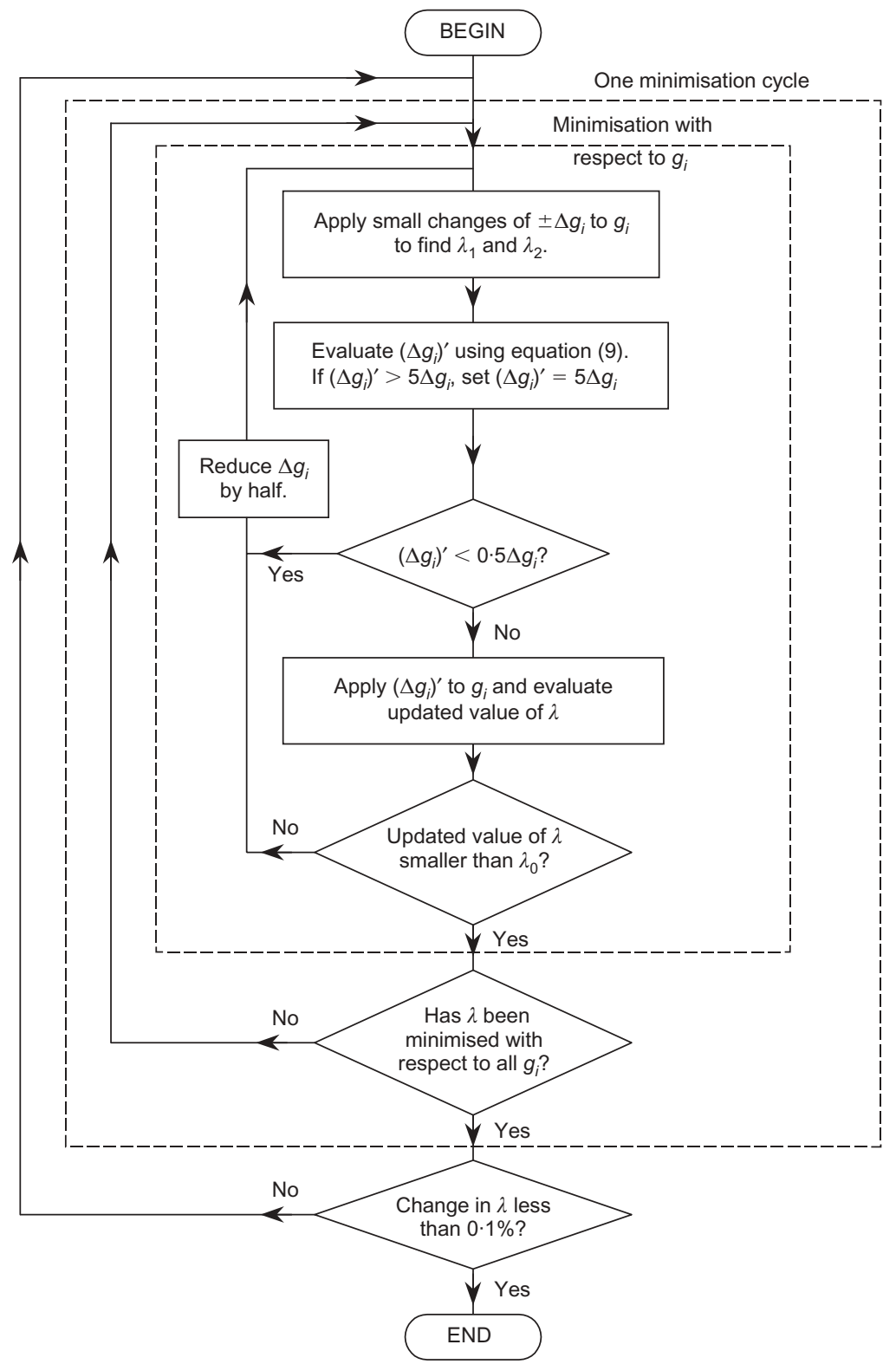

Fig. 6. Procedure for parabolic minimisation

1 min. A flowchart of the minimisation procedure is presented in Fig. 6.

\section{Application to simple cases}

A computer program based on the above numerical procedures has been developed. To verify the correctness of the computer program, a number of examples given in the literature are analysed and compared with the load factors given by others. The examples analysed are shown in Fig. 7.

Example 1 is an orthotropically reinforced rectangular slab simply supported on three sides and free on the fourth, subjected to a uniformly distributed load. It has been analysed by Jones and Wood using hand calculation in chapter 3 of Reference 5 . The computer program yields a load factor equal to $19.06 \mathrm{M} / U L^{2}$, whereas the value of load factor obtained by Jones and Wood is $19 M / U L^{2}$ (to two significant figures). These two results agree closely with each other. In fact, the author has reevaluated the load factor to four significant figures using the formula given by Jones and Wood (equation 3.126) and obtained a value of $19.06 \mathrm{M} / U L^{2}$.

Example 2 is an orthotropically reinforced rectangular slab simply supported on three sides and clamped on the fourth, subjected to a uniformly distributed load. It has been analysed by Jones and Wood using hand calculation in chapter 4 of Reference 5 . The computer program yields a load factor equal to $0.1514 \mathrm{M} / \mathrm{U}$, whereas the value of load factor obtained by Jones and Wood is $0.150 \mathrm{M} / \mathrm{U}$ (to three significant figures). The agreement is considered good. Nevertheless, the author has re-evaluated the load factor to four significant 


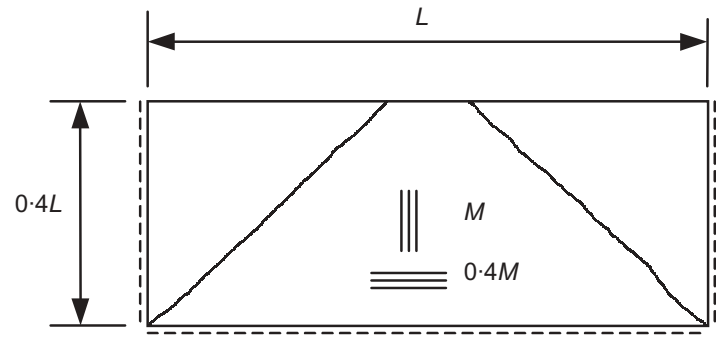

Intensity of u.d.I. $=U$

(a)

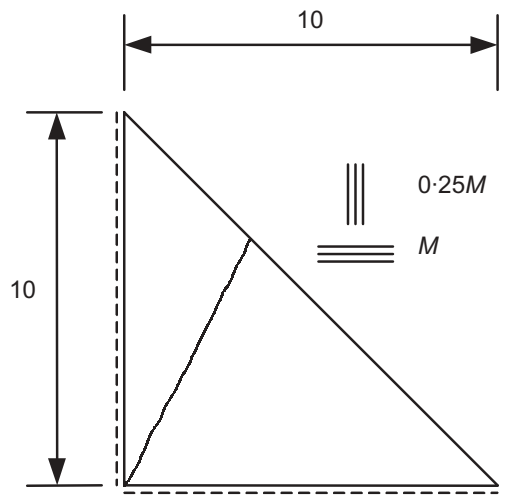

Intensity of line load $=H$

(c)

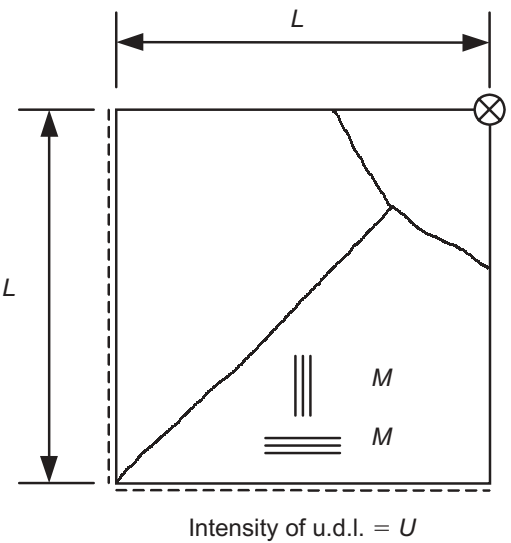

(e)

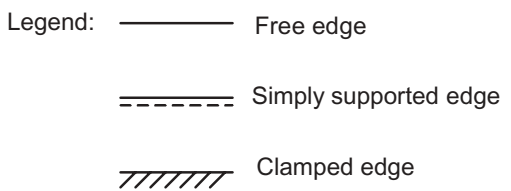

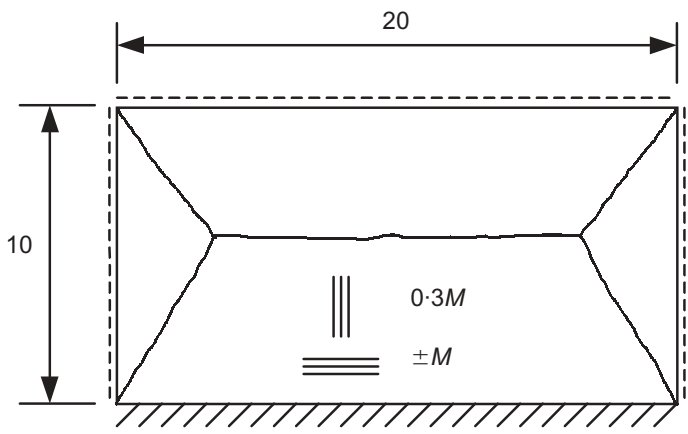

Intensity of u.d.I. $=U$

(b)

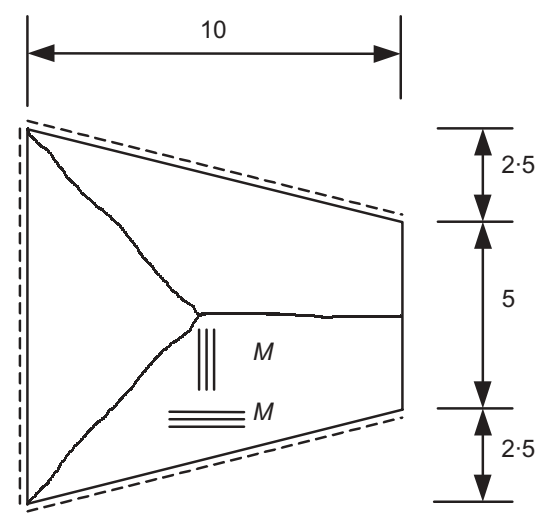

Intensity of u.d.I. $=U$

(d)

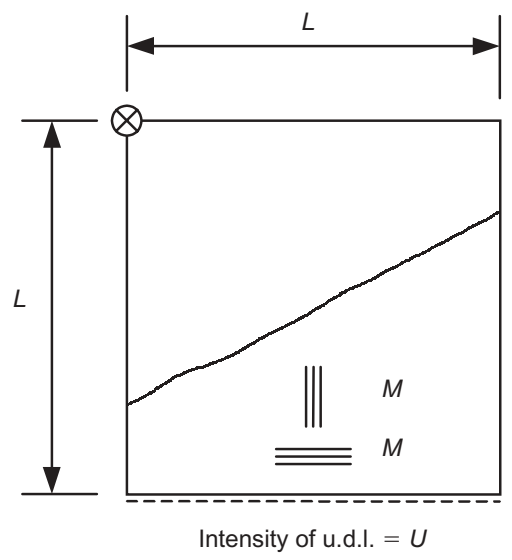

(f)

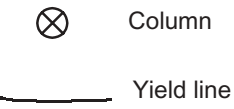

Fig. 7. Application to simple cases: (a) Example 1; (b) Example 2; (c) Example 3; (d) Example 4; (e) Example 5; (f) Example 6

figures using the analytical formulas given by Jones and Wood (equations 4.56, 4.59 and 4.62) and obtained a value of $0 \cdot 1514 \mathrm{M} / U$.

Example 3 is an orthotropically reinforced triangular slab simply supported on two sides and free on the Magazine of Concrete Research, 2004, 56, No. 8 third, subjected to a line load along the free edge. It has been analysed by Jones and Wood using the affinity theorem in chapter 7 of Reference 5. The computer program yields a load factor equal to $0 \cdot 1414 \mathrm{M} / \mathrm{H}$, whereas the value of load factor obtained by Jones and 
Wood is $0 \cdot 141 \mathrm{M} / \mathrm{H}$ (to three significant figures). Very good agreement is achieved. The author has repeated Jones and Wood's calculations with four significant figures retained at all times and obtained a load factor of $0 \cdot 1414 \mathrm{M} / \mathrm{H}$.

Example 4 is an isotropically reinforced trapezoidal slab simply supported on three sides and free on the fourth, subjected to a uniformly distributed load. This example has been analysed by Thavalingam et al. ${ }^{15}$ using a combination of finite-element meshing, linear programming and geometric optimisation. The computer program yields a load factor of $0 \cdot 2872 \mathrm{M} / \mathrm{U}$, whereas the load factor obtained by Thavalingam et al. is $0 \cdot 2850 \mathrm{M} / \mathrm{U}$. The load factor obtained by Thavalingam et $a l$. is slightly smaller and should be more accurate because the corner lever effect has been considered in their solution, whereas in the numerical procedure developed herein the corner lever effect has not yet been taken into account. Nevertheless, the difference in load factor is very small, and the two results may be considered to be in good agreement.

In Example 5 an isotropically reinforced square slab supported on two simply supported edges and one column is analysed. There are two free edges between the column and the supported edges. The slab is subjected to a uniformly distributed load over the whole slab area. It has been analysed by Johansen ${ }^{4}$ using hand calculation. The computer program yields a load factor of $10.67 \mathrm{M} / U L^{2}$, and the load factor obtained by Johansen is also $10 \cdot 67 \mathrm{M} / \mathrm{UL}^{2}$. Exact agreement is achieved.

In Example 6 an isotropically reinforced square slab is supported on one simply supported edge and one column. Apart from the simply supported edge, all the other edges are free. The slab is subjected to a uniformly distributed load over the whole slab area. It has been analysed by Jones and Wood using hand calculation in chapter 6 of Reference 5 . The computer program yields a load factor of $5 \cdot 211 M / U L^{2}$, whereas the load factor obtained by Jones and Wood is $5 \cdot 17 \mathrm{M} / \mathrm{UL}^{2}$ (to three significant figures). As the agreement does not appear to be good, the author has re-evaluated the load factor to four significant figures using the formulae given by Jones and Wood (equations 6.41 and 6.43) and obtained a value of $5 \cdot 211 \mathrm{M} / U L^{2}$.

\section{Application to complicated cases}

In order to demonstrate the versatility of the computer program, two more complicated examples are analysed, as shown in Fig. 8.

Example 7 is a five-sided polygonal slab supported on two clamped edges and two columns. There are three free edges between the clamped edges and the columns. The slab is isotropically reinforced with plastic moment $M$ under both sagging and hogging conditions, and is subjected to a uniformly distributed load of $U$. The number of minimisation cycles required to complete the analysis is six, and the minimum load factor obtained is $0 \cdot 1967 \mathrm{M} / \mathrm{U}$.

Example 8 is another five-sided polygonal slab supported on one clamped edge, two simply supported edges and one column. There are two free edges between the column and the supported edges. The slab is orthotropically reinforced with plastic moment $0.5 \mathrm{M}$ about the $x$-axis and plastic moment $M$ about the $y$ axis. Equal amounts of reinforcement are provided at the top and bottom of the slab, and thus the moment capacities of the slab under sagging and hogging conditions are the same. A point load of magnitude $F$ and a patch load of magnitude $10 F$ evenly distributed over an area of $2 \times 2$ are applied to the slab, as shown in the diagram. The number of minimisation cycles required to complete the analysis is 10 , and the minimum load factor obtained is $0.2325 \mathrm{M} / \mathrm{F}$.

\section{Discussion}

The numerical procedure developed herein has the major limitation that it is at present applicable only to polygonal-shaped slabs with no concave corners. Yield line analysis of polygonal-shaped slabs with concave corners can be quite complicated, for the following reasons: (a) there may be more than one slab region associated with a point support at a concave corner; and (b) there will be both positive and negative yield lines within the slab area, rendering the identification of yield lines from the intersections between adjacent slab regions much more difficult. Nevertheless, it is believed that the same principle of using dip and strike angles to define all yield line patterns can still be applied. Further research to extend the dip and strike angles method to polygonal-shaped slabs with concave corners is recommended.

There is also the limitation that, like Jennings' theorem, which associates each slab region with a supported edge, ${ }^{17}$ the proposed methodology of defining the yield line pattern in terms of the dip and strike angles of the slab regions, each associated with a support, is strictly applicable only to the areas of slab that take part in the collapse mechanism - that is, the areas that do deflect. In reality there are cases in which the collapse mechanism does not encompass the whole area of the slab. Examples are when corner levers are present, or when there are circular fan mechanisms. In such cases the proposed methodology should be restricted to the areas that do deflect. Nevertheless, the dip and strike angles method may be extended to deal with these cases by introducing additional geometric parameters to define the boundaries of the deflected areas and applying the same parabolic minimisation procedure to determine the values of the additional parameters. The author has tried to take corner lever effects into account by introducing the aforesaid addi-

Magazine of Concrete Research, 2004, 56, No. 8 


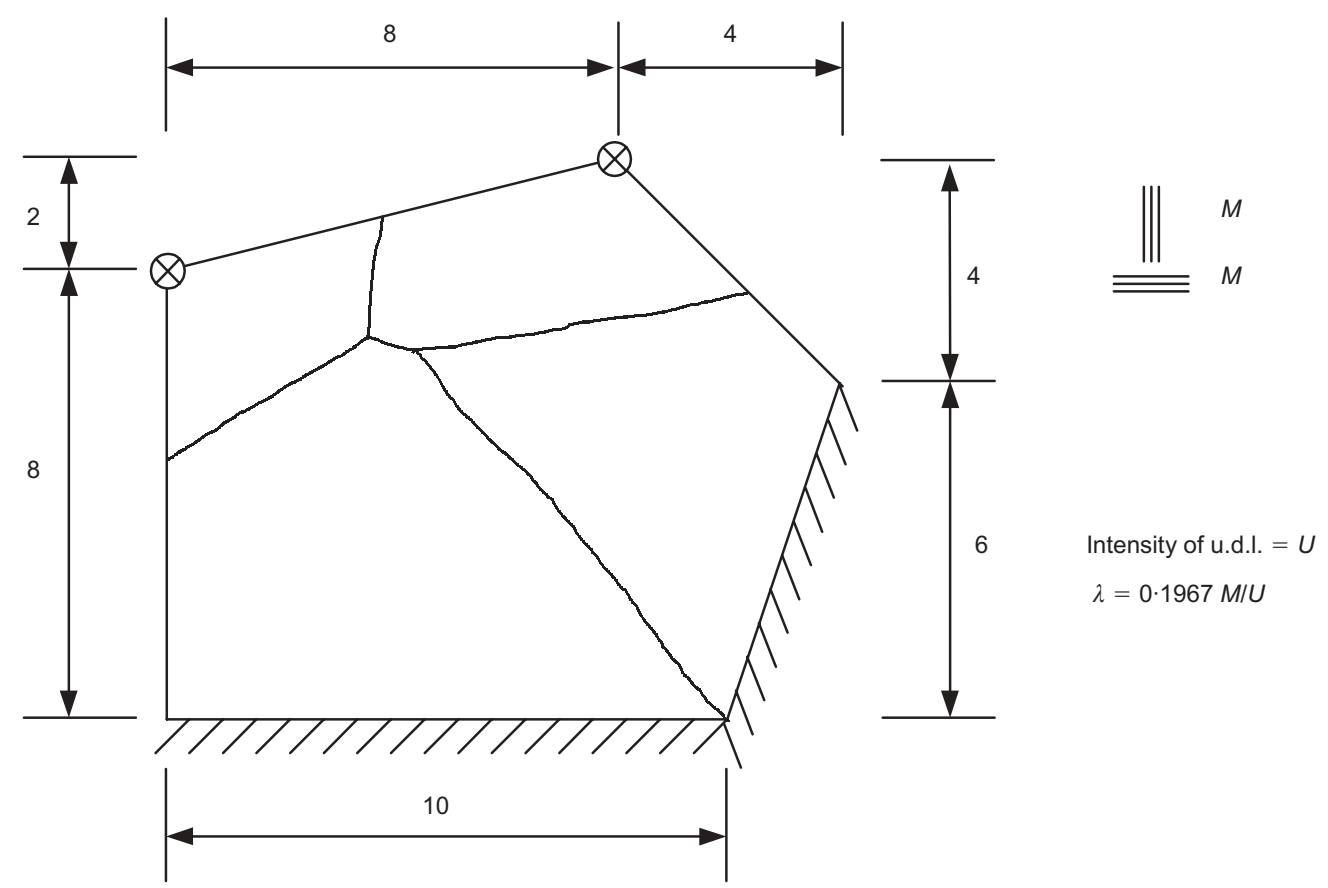

(a)

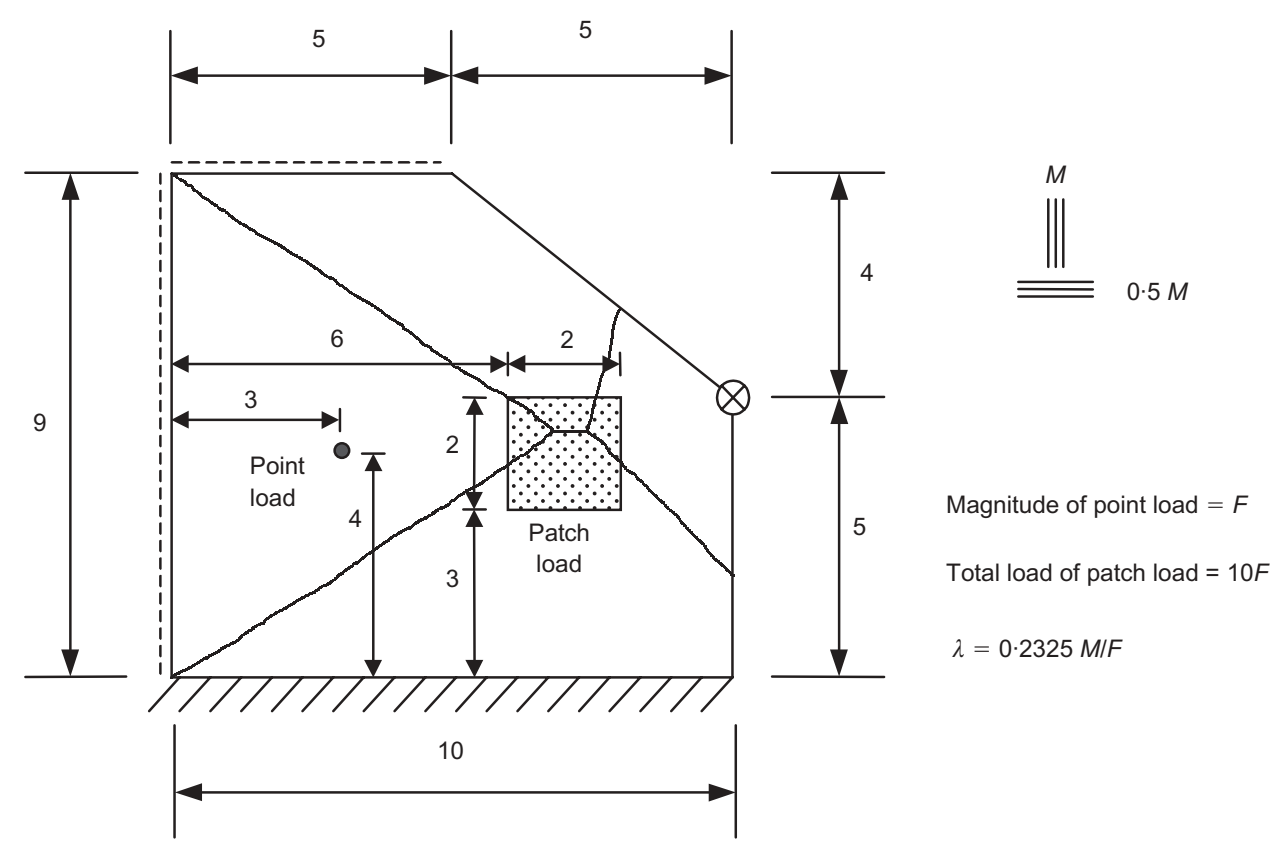

(b)

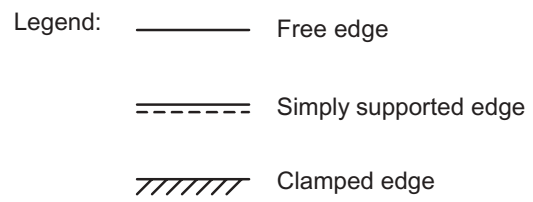

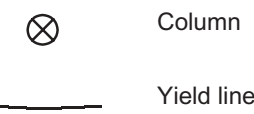

Fig. 8. Application to complicated cases: (a) Example 7; (b) Example 8 
tional parameters, and has obtained very promising results. Further work to deal with circular fan mechanisms is still under way. The results will be presented in a later paper when completed.

\section{Conclusions}

A new yield line analysis method that defines the collapse mechanism and yield line pattern in terms of the dip and strike angles of the deflected surfaces of the slab regions separated by the yield lines has been developed. As there is a one-to-one correspondence between any kinematically admissible yield line pattern and the set of geometric parameters comprising the dip and strike angles, this method has the major advantages that any yield line pattern defined in terms of the dip and strike angles is always kinematically admissible, and that it is possible to generate all the kinematically admissible yield line patterns to be considered in the yield line analysis by just varying the values of the dip and strike angles.

The required numerical procedures for the yield line analysis, including the generation of yield line pattern from a given set of dip and strike angles, and evaluation of the external work done, internal energy dissipation along yield lines and load factor as functions of the dip and strike angles, have been derived. The final solution is obtained by minimising the load factor with respect to the dip and strike angles. As there could be slope discontinuities, the minimisation cannot be carried out using any numerical method that relies on gradients. To overcome this difficulty, a new minimisation technique based on successive parabolic minimisation that is applicable even when there is slope discontinuity has been developed.

A computer program based on the above methodologies has been produced. It is fully automatic in the sense that the user no longer needs to input any assumed yield line pattern that is kinematically admissible. Its correctness has been verified by checking against results obtained by others for simple cases, and its versatility has been demonstrated by applying it to more complicated cases. However, there are still the limitations that it is not applicable to slabs with concave corners, and that the corner lever effect is not yet taken into account. Nevertheless, it is believed that the general principles proposed herein should be applicable to arbitrarily shaped slabs, and that it is only a matter of further detailed studies to have the dip and strike angles method extended to cover all slab configurations, including those with concave corners, and to incorporate the corner lever effect.

\section{Acknowledgements}

The financial support of the Croucher Foundation for the research work presented herein is gratefully acknowledged. Two of the author's students, Mr Y. K. Lam and Mr Ivan Y. T. Ng, have helped to work out the numerical examples and to draw the diagrams.

\section{References}

1. Kong F. K. and Evans R. H. Reinforced and Prestressed Concrete, 3rd edn. Van Nostrand Reinhold, Wokingham, 1987, pp. 292-332.

2. Park R. and Gamble W. L. Reinforced Concrete Slabs, 2nd edn. John Wiley \& Sons, New York, 2000.

3. Ingerslev A. The strength of rectangular slabs. Journal of Institution of Structural Engineers, 1923, 1, No. 1, 3-14.

4. Johansen K. W. Brudlinieteorier, Copenhagen, 1943 (in Danish). English translation: Yield Line Theory, Cement and Concrete Association, London, 1962.

5. Jones L. L. and Wood R. H. Yield Line Analysis of Slabs. Thames \& Hudson, London, 1967.

6. Mills G. M. A partial kinking yield criterion for reinforced concrete slabs. Magazine of Concrete Research, 1975, 27, No. 90, 13-22.

7. Simmonds S. and Ghali A. Yield-line design of slabs. Journal of Structural Division, ASCE, 1976, 102, No. ST1, 109-123.

8. Bauer D. and Redwood R. G. Numerical yield line analysis. Computers \& Structures, 1987, 26, No. 4, 587-596.

9. Dickens J. G. and Jones L. L. A general computer program for the yield-line solution of edge supported slabs. Computers \& Structures, 1988, 30, No. 3, 465-476.

10. Denton S. R. Compatibility requirements for yield-line mechanisms, Solids \& Structures, 2001, 38, 3099-3109.

11. Munro J. and Da Fonseca A. M. A. Yield line method by finite elements and linear programming. Structural Engineer, 1978, 56B, No. 2, 37-44.

12. Jennings A., Thavalingam A., McKeown J. J. and Sloan D. On the optimisation of yield line patterns. In Developments in Computational Engineering Mechanics (TopPING B. H. V. (ed.)). Civil-Comp Press, Edinburgh, 1993, pp. 209-214.

13. Johnson D. Mechanism determination by automated yield-line analysis. Structural Engineer, 1994, 72, No. 19, 323-327.

14. Johnson D. Yield-line analysis by sequential linear programming. Solids \& Structures, 1995, 32, No. 10, 1395-1404.

15. Thavalingam A., Jennings A., McKeown J. J. and Sloan D. A computerised method for rigid-plastic yield-line analysis of slabs. Computers \& Structures, 1998, 68, 601-612.

16. Thavalingam A., Jennings A., Sloan D. and McKeown J. J. Computer-assisted generation of yield-line patterns for uniformly loaded isotropic slabs using an optimisation strategy. Engineering Structures, 1999, 21, 488-496.

17. Jennings A. On the identification of yield-line collapse mechanism. Engineering Structures, 1996, 18, No. 4, 332-337.

18. Blyth F. G. H. and De Freitas M. H. A Geology for Engineers, 6th edn. English Language Book Society and Edward Arnold (Publishers), London, 1974.

Discussion contributions on this paper should reach the editor by 1 April 2005 